\title{
Autonomous Driving Path Planning based on Sarsa-Dyna Algorithm
}

\author{
Aboul Ella Hassanien1), Jennefer Mononteliza2)
}

\begin{abstract}
In autonomous driving path planning, ensuring the computational efficiency and safety of planning is an important issue. The Dyna framework in reinforcement learning can solve the problem of planning efficiency. At the same time, the Sarsa algorithm in reinforcement learning can be effective in guaranteeing the safety of path planning. This paper proposes a path planning algorithm based on Sarsa-Dyna for autonomous driving, which effectively guarantees the efficiency and safety of path planning. The results show that the number of steps planned in advance is proportional to the convergence speed of the reinforcement learning algorithm. The Sarsa-Dyna will be proposed. The analysis of convergence speed and collision times has been done between the proposed Sarsa-Dyna, Q-learning, Sarsa and Dyna-Q algorithm. The proposed Sarsa-Dyna algorithm can reduce the number of collisions effectively, ensure safety during driving, and at the same time ensure convergence speed.
\end{abstract}

Keywords: Autonomous Driving, Reinforcement, Learning, Path Planning

\section{Introduction}

In autonomous driving, path planning is a very important issue. At this stage, good urban traffic path planning can ensure the safety of autonomous driving and improve efficiency. The standardized definition of urban traffic path planning is as follows: Specify a vehicle as an agent, the purpose is to allow the agent to find a barrier-free path from the initial state to the target state from the state within the specified range. How to improve the efficiency of the path planning algorithm has become an urgent problem to be solved. The path planning algorithm uses an efficient planning algorithm to select a reasonable navigation path for the intelligent vehicle. This reduces the time the vehicle runs on the city road. And then the patency of the city road will be improved.

At present, the problem of path planning has attracted the attention of many scholars, and

Received(March 18, 2020), Review Result(1st: April 14, 2020, 2nd: June 02, 2020), Accepted(June 25, 2020)

1) (Professor, Corresponding Author) Information Technology Department, Cairo University, Egypt email: abo@egyptscience.net

2) (Professor) University of Antique, Antique, Philippines

email: jenneferjmonteliza@gmail.com 
there are some successful research results, which can be divided into global planning and partial planning. The algorithms for global planning include the $A^{*}$ algorithm[1][2] and its various improved versions, etc. These algorithms have performed excellent results in offline static environments.

For dynamic environments, $\mathrm{Yu}$ et al. proposed a distributed algorithm (k Shortest Path in Dynamic Graphs, KSP-DG) for identifying k-shortest paths in dynamic graphs, which can find multiple shortest paths in dynamic road network environments[3][4]. However, these algorithms rely too much on environmental data, and require a large amount of data to support path planning, and the effect is poor in an unknown environment, and they cannot be applied to environmental changes. Partial planning includes neural network method[5], genetic algorithm and reinforcement learning algorithm. Among them, reinforcement learning algorithms have been widely used because they do not require prior knowledge of the environment[6].

By applying the reinforcement learning algorithm to the path planning algorithm, it can effectively help the vehicle's travel path planning. At the same time, the changes in the road traffic state in the future can be considered, which can further improve the rationality of the routing algorithm. It is important for alleviating urban traffic congestion.

This paper proposes a vehicle path planning algorithm based on reinforcement learning in a road traffic network environment. The Dyna framework that combines model-based algorithms and model-independent algorithms can increase the speed of planning. At the same time, the more classic Sarsa algorithm is used as a road strategy that can improve the security of the algorithm[7].

Compared with other existing algorithms, the path planning algorithm based on reinforcement learning has the following characteristics:

(1) Most of the path planning environment is unknown, and the algorithm needs to be built on the model before the path search. The model-independent algorithm can work in an unknown environment, the algorithm is adaptive.

(2) Some path planning algorithms can find the optimal route, but it may take more time, which may cause a waste of resources. Reinforcement learning algorithms can find a relatively fast and better route, and at the same time improve the safety of path planning.

(3) Reinforcement learning is a process of constant experimentation. Find the optimal strategy by constantly interacting with the environment. The strategy generated in this way must be complete for the smart car. It will not be impossible to execute because the path is too complicated.

Simulation experiments show that, compared with the classic path planning algorithm, the 
path planning algorithm based on reinforcement learning can truly simulate the actual driving state of intelligent vehicles, effectively reduce the travel time overhead of vehicles in the environment, and improve the efficiency of urban traffic operation.

\section{Related Works}

Among the reinforcement learning algorithms to solve the path planning problem, the Q-Learning algorithm[8] is a more commonly used algorithm.

Qiao Junfei et al.[9] introduced BP the neural network to the Q-learning algorithm and applied it to mobile robots, which can realize path planning for unknown environments.

Wang et al.[10] combined the Q-learning algorithm with the Sarsa algorithm and proposed a reverse Q-learning algorithm.

Gosavi[11] proposed a path planning method based on Q-learning and neural network planners. The experiment proved the effectiveness of this method in an environment with known global information.

Sarsa algorithm is a model-independent reinforcement learning method based on the $Q$ learning algorithm. The path planning algorithm based on Sarsa has also been researched.

The combination of the Sarsa algorithm and other methods has also achieved certain research results, such as the k-means clustering algorithm (k-means)[12] and neural network algorithm[13-15].

The algorithms in these documents can complete the basic requirements of path planning, but the convergence speed is slow and it takes a lot of time. In 1991, Richard Sutton, a pioneer in reinforcement learning, developed the Dyna framework. This framework can effectively improve the convergence speed of the algorithm by adding the experience gained from interacting with the simulated environment. The Dyna framework is usually used in conjunction with Q-learning algorithms. However, a problem with the Q-learning algorithm is that excessive pursuit of reward maximization causes the agent to fall into danger too many times in the barrier map.

\section{Method}

Algorithm theory involves the classic Markov Decision Process (MDP), which is described by the four-tuple $<\mathrm{S}, \mathrm{A}, \mathrm{P}, \mathrm{R}>$, where

$S$ represents the set of states and refers to the location. 
A represents the set of actions that the agent can take. The agent car can perform 4 actions in the grid, which are up, down, left, and right.

$\mathrm{P}$ represents the state transition probability matrix, representing the probability of the agent car choosing up, down, left, and right

$\mathrm{R}$ represents the reward function. When the agent moves in the simulated map, the reward value is 0 when it does not collide with a barrier, the reward value is -1 when it collides with a barrier, and the reward value is 2 when it moves to the target point.

Markov decision process provides a basic theoretical framework for reinforcement learning problems. Almost all reinforcement learning problems in the existing environment can be modeled using MDP.

\subsection{Model-independent reinforcement learning algorithm}

Sarsa algorithm is a method of solving reinforcement learning control problems using a time series difference. It is similar to the Q-learning algorithm. It uses the Q-table method to store the action-value function, and the decision-making part uses the greedy strategy (epsilon-greedy) to select an action. Sarsa algorithm is an online learning algorithm. It will execute the action first and then update it. The basic update rule is as shown in formula (1):

$$
Q(S, A)=Q(S, A)+a\left(R+\gamma Q\left(S^{\prime}, A^{\prime}\right)-Q(S, A)\right)
$$

where $S$ is the current state, $A$ is the action selected in the current state, $S^{\prime}$ is the next state, $\mathrm{A}^{\prime}$ is the action selected in the next state, and $\mathrm{R}$ is the reward value, $\mathrm{\gamma}$ is the discount factor, $\mathrm{a}$ is the learning factor, and $\mathrm{Q}$ is the set of $\mathrm{q}$ values. In the simulation experiment, the learning rate $\mathrm{a}=0.01$ and the discount factor $\mathrm{\gamma}=0.9$.

It can be seen from the update function. Compared with the Q-learning algorithm, the Sarsa algorithm is more conservative algorithm. It cares more about the decision-making at each step. It is more sensitive to errors and traps. In urban traffic path planning, smart cars are more sensitive to collisions, so the system needs a safer algorithm to guide the movement of smart cars. The Sarsa algorithm is a model-free and strong chemical algorithm that takes into account safety and speed. It is more in line with the urban traffic environment, so the Sarsa algorithm is chosen as the basic algorithm to guide the path planning of the agent. 
In the model-independent reinforcement learning algorithm, the agent can use the experience generated in the environment to directly learn the strategy[16]. while in the model-based reinforcement learning, the agent uses the simulation experience generated by the simulation to plan a new strategy. In the Dyna framework, the agent uses the direct experience generated by the interaction with the environment to learn a model, and at the same time uses the model to generate simulated experience, uses real experience and simulated experience to act on the agent at the same time, to help the agent learn and plan a suitable strategy.

Dyna framework is shown in [Fig. 1].

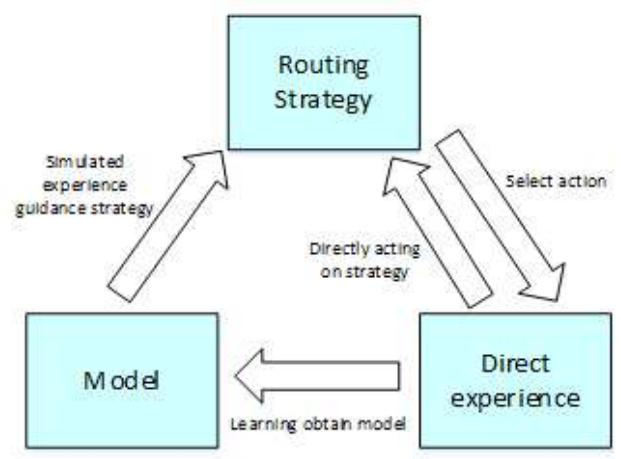

[Fig. 1] Dyna Framework

From [Fig. 1], the Dyna framework represents a planning process. Each time the agent finds a path, the Dyna framework will guide the choice of the agent through the acquired simulation experience and perfect model, so that the agent can continue to think during the operation and choose the optimal path faster. Compared with other algorithms, convergence speed has certain advantages. In urban traffic path planning, the vehicle is used as an agent, the position where the vehicle can move is used as the state space, and each action selected by the vehicle is used as the action space. When the vehicle selects the path to the target location, the vehicle first selects the next state in the state space according to the value of the reward function, and at the same time constructs a map of the environment in the system[17]. The vehicle continues to interact with the real environment to gain direct experience, and the vehicle also interacts with the built model to gain simulation experience to help the smart car plan its route in advance. Under the combined effect of the two, the vehicle can avoid choosing a poor path and improve the speed of vehicle path planning. 


\section{Dyna Framework Based Sarsa Algorithm}

Dyna framework can be combined with a variety of reinforcement learning algorithms.

The combination method is to use the basic algorithm to interact with the unknown environment to obtain real samples, then use the Dyna framework to plan multiple times on the map model established in the interaction to obtain virtual samples, and finally use the real samples and virtual samples to jointly guide the agent's path planning process.

In the proposed Sarsa-Dyna method, the basic algorithm is the Sarsa algorithm. First of all, Sarsa algorithm is an online learning algorithm, it will learn in the process of state transition, and the entire learning and action process uses only one strategy, and uses an e-greedy method with two times to select $Q(S, 4)$ and $Q\left(S^{\prime}, A^{\prime}\right)$. Compared with other algorithms, the Sarsa algorithm is more cautious. Combined with the planning process of the Dyna learning framework, it not only ensures the safety of the algorithm, but also improves the running speed of the algorithm.

Sarsa-Dyna used in the smart vehicle is shown in [Fig. 2].

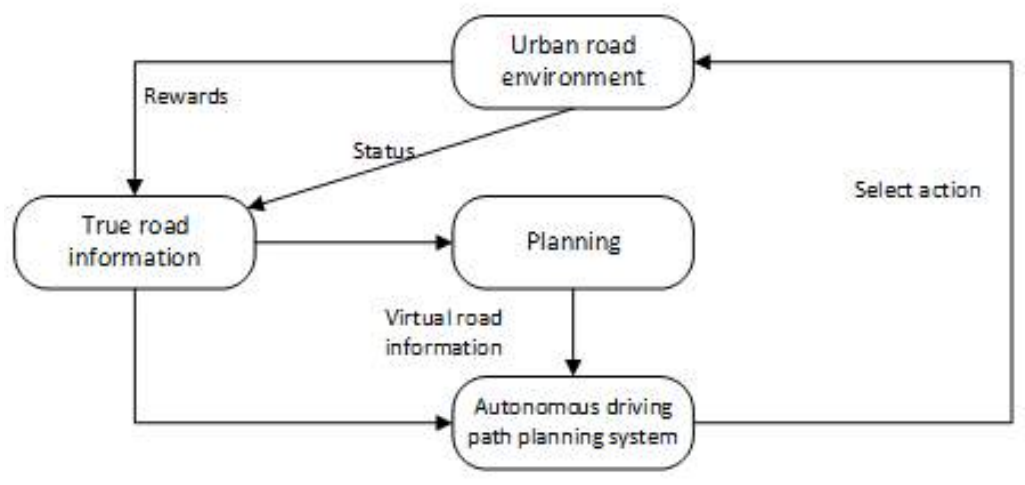

[Fig. 2] Sarsa-Dyna Framework

Combining the Sarsa algorithm with the Dyna framework, the purpose is to apply the Sarsa-Dyna algorithm to urban path planning, as follows:

The research problem is single agent path planning, and the agent is a controlled vehicle.

In the process of realizing path planning, the agent needs to find a path according to some specific rules. These specific rules are algorithms. The Sarsa-Dyna algorithm is applied as a new rule to the urban path planning problem.

In urban route planning, with the operation of vehicles, the environment in which they are 
located is constantly changing, which is a great challenge for path planning.

http://dx.doi.org/10.47116/apjcri.2020.07.06

The Dyna framework can solve this problem well. The agent obtains both simulation experience from the model and experience from the direct interaction environment. The two kinds of experience can provide guidance for the agent to choose a route. The agent can quickly find a safe path.

The following are the steps of the Sarsa-Dyna algorithm:

(1) Initialize the model to understand Model (s, a),

(2) For each round of iteration, first obtain a new state $S^{\prime}$ and reword $R$ according to the current state and $\mathrm{Q}(\mathrm{S}, \mathrm{A})$ in a $\varepsilon$-greedy way.

(3) Then update the value function $Q(S, A)$ with Sarsa

(4) Update the model Model(s, a) with $\mathrm{R}, \mathrm{S}^{\prime}$ (After interacting with the real environment, perform $\mathrm{n}$ simulations)

(5) Randomly select one for each simulation state $S$ that has appeared before, and based on this, an action $\mathrm{A}$ is randomly selected.

(6) Based on the Model (S, A) to get $S^{\prime}$ and $R$

(7) Then use Sarsa to update the value function

In the basic process of the Sarsa-Dyna algorithm, the external cycle process directly uses data experience to build the environment model to update the value function, and the internal cycle process uses the construction model to generate new simulation experience to update the value function. Compared to the simple planning process or reinforcement learning algorithm, the Sarsa-Dyna algorithm shows higher efficiency.

Sarsa-Dyna is an algorithm to ensure safety. Its security depends on the cooperation of basic algorithms and frameworks. The basic algorithm Sarsa uses the idea of generalized strategy iteration. It will use the next state $S^{\prime}$ and the $\mathrm{Q}$ value of the action $\mathrm{A}^{\prime}$ given by the state to estimate the target value. This will be more sensitive to the choice of the target value, and the agent will choose to stay away from the state where the reward value is low. At the same time, the Dyna framework is used for continuous simulation operation, and risky behaviors can be recorded during the operation. The agent can avoid these paths and choose the path with higher security.

In terms of speed, Sarsa-Dyna uses the Dyna framework to directly model the environment. The algorithm obtains both simulation experience and direct interaction experience, which is equivalent to the agent using less time to try more paths in an unknown environment. Compared with other algorithms, it can help agents choose the optimal path faster. Finally, the convergence speed of the entire algorithm is effectively improved. 


\section{Simulation Experiment}

Use $\mathrm{Q}$ learning algorithm, Sarsa algorithm and Dyna-Q algorithm as comparative experiments to compare with the new algorithm.

The relevant parameters of all experiments are as follows: the maximum trial round of the algorithm is set to 100 , the learning rate $a$, the discount factor $\gamma$, and the greedy value $r$.

The learning rate, discount factor, and greedy value need to be obtained by running experiments to adjust the parameters. The experiment is as follows.

The first adjustment is the learning rate of the reinforcement learning algorithm. The learning rate can be understood as the learning efficiency of the algorithm. When the learning rate is large, the learning speed of the algorithm is fast, but it is easy to cause the easy loss value to explode; when the learning rate is small, the learning speed of the algorithm is slow, and at the same time, over-fitting problems will occur, and the convergence speed of the algorithm will slow down.

Since the simulation environment is relatively simple, there is no need to set a dynamically changing learning rate to make the algorithm achieve optimal results.

Through experiments we can get: when the learning rate is less than 0.01 , the algorithm convergence speed is too slow, which affects the performance of the algorithm. When the learning rate is greater than 0.01 , the gradient may oscillate around the minimum value, and even fail to converge; when the learning rate is equal to 0.01 , the convergence speed is optimal, and there are no other side effects. The learning rate of the algorithm is set to 0.01 .

Then adjust the discount factor to find the optimal performance. The discount rate can avoid an infinite loop of states. When the discount rate is small, the agent is more concerned about short-term returns; when the discount rate is large, long-term returns become more important. Through experiments, it is concluded that when the discount factor is 0.9 , the convergence speed can be ensured, and the non-convergent variance will not be caused, which is more suitable for the simulation experiment map environment.

As the Sarsa algorithm is used as the basic algorithm, the sampling strategy and the guiding strategy are both greedy strategies, which involves the selection of greedy values. Therefore, the optimal greedy value needs to be obtained by designing experiments to ensure the performance of the algorithm, and other parameters remain unchanged during the change of the greedy value. During the experiment, the greedy degree takes a value in the interval $(0,1)$. Some classic literature divides the greedy value into three intervals, namely $(0,0.1),(0.1,0.2),(0.2,1)$. 
Three Dyna-Sa algorithms with different degrees of greed are applied to the barrier map, and 100 iterations of training are performed, and the convergence speed and the number of collisions are used as the evaluation criteria.

Finally, the following results can be obtained as [Table 1].

[Table 1] Performance of Three Greed Degree

\begin{tabular}{|c|c|c|}
\hline Greedy degree & Convergence speed & collisions \\
\hline$(0,0.1)$ & 13.6 & 65 \\
\hline$(0.1,0.2)$ & 15.4 & 31 \\
\hline$(0.2,1)$ & - & 48 \\
\hline
\end{tabular}

It can be seen from [Table 1] that when the greedy degree is in the interval of $(0.1,0.2)$, the algorithm can show the best performance. When the greedy degree is in the range of (0.0.1), the algorithm will choose the state with the largest reward value, so the convergence speed is slower, and there are more collisions, and the safety is poor. When the greedy degree is in the range of $(0.2,1)$, the algorithm performance is too conservative, and the agent will stagnate in a certain safe area, causing the algorithm to fail to converge, and there are more collisions.

Finally, the learning rate is set to 0.01 , the discount factor is set to 0.9 , the greed value is set to 0.2 in the barrier map of the simulation experiment, and 0.1 in the barrier-free map.

The simulation map environment is relatively simple, and the value of the reward $\mathrm{R}$ has limited influence on the experimental results, so the algorithm function can be realized by choosing a simple value. When the agent moves in the simulated map, the reward value is 0 when it does not collide with a barrier, the reward value is -1 when it collides with a barrier, and the reward value is 2 when it moves to the target point.

According to the parameter settings above, the four algorithms were introduced into the map for experiments. Each algorithm performs 100 iterations of training, and the number of planning using the Dyna framework is 30, which means that the agent interacts with the simulation environment 30 times to obtain virtual experience. Each algorithm experiment is repeated 10 times, and then the average value is taken as the experimental output data. The evaluation criteria of algorithm performance are the average running time of the algorithm, the average number of collisions with barriers, the rounds required to achieve convergence and the time required to achieve convergence. The experimental results of barrier-free maps and barrier-free maps are shown in [Table 2] and [Table 3]. 
[Table 2] Barrier-free Map Results

\begin{tabular}{|c|c|c|c|}
\hline Algorithm & Average run time & $\begin{array}{c}\text { Time of reaching } \\
\text { convergence }\end{array}$ & $\begin{array}{c}\text { Rounds of reaching } \\
\text { convergence }\end{array}$ \\
\hline Q-Learning & 264 & 105.7 & 16 \\
\hline Sarsa & 253 & 106.8 & 5 \\
\hline Dyna-Q & 104 & 11.2 & 5 \\
\hline Sarsa-Dyna & 118 & 13.3 & \\
\hline
\end{tabular}

[Table 3] Barrier Map Results

\begin{tabular}{|c|c|c|c|c|}
\hline Algorithm & Average run time & Average collision & $\begin{array}{c}\text { Time of } \\
\text { reaching } \\
\text { convergence }\end{array}$ & $\begin{array}{c}\text { Rounds of } \\
\text { reaching } \\
\text { convergence }\end{array}$ \\
\hline Q-Learning & 343 & 87 & 141.5 & 38 \\
\hline Sarsa & 682 & 76 & 236.7 & 128 \\
\hline Dyna-Q & 119 & 29 & 23 & 34 \\
\hline Sarsa-Dyna & 176 & 20 & 19 & 19 \\
\hline
\end{tabular}

From the data in [Table 2] and [Table 3], it can be seen that the data of the algorithm using the Dyna framework is better than the algorithm without the framework, which reflects the efficiency of planning.

In the experiment of barrier-free maps, the performance of the Dyna-Q algorithm and the Sarsa-Dyna algorithm is similar.

In the experiment with barrier maps, the Sarsa-Dyna algorithm is better than the Dyna-Q algorithm in terms of the average number of collisions, convergence rounds and convergence time, but Sarsa-Dyna performs slightly worse in terms of average total running time.

The reason is that the Sarsa-Dyna algorithm is a risk-sensitive algorithm. At the early stage of learning, the Sarsa-Dyna algorithm abandons the closer path to avoid barriers to ensure safety, but it will lead to a waste of time. The Sarsa algorithm is the same.

After multiple learning, the experience gained by the Sarsa-Dyna algorithm will guide the agent to choose the optimal path, and the time required will gradually shorten.

From the data in the comprehensive table, it can be concluded that the Sarsa-Dyna algorithm is better than the other three algorithms on the whole. While ensuring safety, it can find a better route in a short time to meet the special requirements of autonomous driving route 
planning[18].

\section{Conclusion}

Aiming at the problem of urban traffic route planning, the new algorithm Sarsa-Dyna is mainly based on the Dyna framework, using Sarsa's update function and decision-making method, and the Dyna framework that combines model-independent and model-based algorithms to introduce models to model-free reinforcement learning algorithms. Use the learned experience to train the model, and at the same time use the model to generate a simulated experience, and finally, the experience directly learned from the environment works together to update the value function. The paper analyzes the peculiarities of urban traffic route planning, compares the characteristics of the Sarsa algorithm and classical reinforcement learning algorithm Q-learning, and chooses the more conservative and suitable Sarsa algorithm combined with the Dyna framework. Simulation experiments show that the more times the agent plans, the more simulation experience it learns and the faster the algorithm's convergence speed. The results show that the Dyna-s algorithm can effectively avoid barriers, improve the safety of path planning, and at the same time ensure that a path that meets the requirements can be found quickly, which is more suitable for application in autonomous driving path planning.

Future work is mainly to study the performance of the algorithm in a dynamic traffic environment and increase the variable function in the setting of the reward value to improve the overall convergence speed of the algorithm and improve the safety of the vehicle path planning process.

\section{References}

[1] Peter E. Hart, Nils J. Nilsson, Bertram Raphael, A Formal Basis for the Heuristic Determination of Minimum Cost Paths, IEEE Transactions on Systems Science and Cybernetics, (1968), Vol.4, No.2, pp.100-107.

[2] JinYeong Um, A Performance Model of Controller Area Networks for Vehicles under Unsaturated Traffic, International Journal of Reliable Information and Assurance, (2020), Vol.8, No.1, pp.1-6, 10.21742/IJRIA.2020.8.1.01

[3] Ziqiang Yu, Xiaohui, Yu, Nick Koudas, Yang Liu, Yifan Li, Yueting Chen, Dingyu Yang, Distributed Processing of $\mathrm{k}$ Shortest Path Queries over Dynamic Road Networks, The 2020 ACM SIGMOD International Conference on Management of Data, (2020), Jun 14-19; Portland, USA.

[4] N. Thirupathi Rao, Debnath Bhattacharyya, Traffic Supervision System Using Hyg12 Load Sensors, International Journal of Science and Engineering for Smart Vehicles, (2019), Vol.3, No.1, pp.21-30, DOI: 
10.21742/IJSESV.2019.3.1.03

[5] Roy Glasius, Andrzej Komoda, Stan C.A.M. Gielen, Neural Network Dynamics for Path Planning and Obstacle Avoidance, Neural Networks, (1995), Vol.8, No.1, pp.125-133.

[6] Minwoo Ryu, Si-Ho Cha, Developing Multi-Directional Broadcasting for Both Highway and Urban Vehicular Sensor Networks, International Journal of Science and Engineering for Smart Vehicles, (2019), Vol.3, No.1, pp.1-6, 10.21742/IJSESV.2019.3.1.01

[7] Richard S. Sutton, Andrew G. Barto, Reinforcement Learning: An Introduction, UK: MIT Press, (2017)

[8] Chaymaa Lamini, Youssef Fathi, Said Benhlima, H-MAS architecture and reinforcement learning method for autonomous robot path planning, The 2017 Intelligent Systems and Computer Vision (ISCV), (2017), Apr 17-19; Fez, Morocco.

[9] Junfei Qiao, Zhanjun Hou, Xiaogang Ruan, Application of reinforcement learning based on neural network to dynamic obstacle avoidance, The 2008 International Conference on Information and Automation, (2008), Jun 20-23; Changsha, China.

[10] Yin-Hao Wang, Tzuu-Hseng S. Li, Chih-Jui Lin, Backward Q-learning: The combination of Sarsa algorithm and Q-learning, Engineering Applications of Artificial Intelligence, (2013), Vol.26, No.9, pp.2184-2193.

[11] Abhijit Gosavi, Reinforcement Learning: A Tutorial Survey and Recent Advances, Informs Journal on Computing, (2009), Vol.21, No.2, pp.177-345.

[12] M. Andrecut, M. K. Ali, Deep-Sarsa: A Reinforcement Learning Algorithm For Autonomous Navigation, International Journal of Modern Physics C, (2001), Vol.12, No.10, pp.1513-1523.

[13] Hoang Huu Viet, Sang Hyeok An, Tae Choong Chung, Dyna-Q-based vector direction for path planning problem of autonomous mobile robots in unknown environments, Advanced Robotics, (2013), Vol.27, No.3, pp.159-173.

[14] Hao Jiang, Renjie Gui, Zhen Chen, Liang We, Jian Dang, Jie Zhou, An Improved Sarsa( $(\lambda)$ Reinforcement Learning Algorithm for Wireless Communication Systems, IEEE Access, (2019), Vol.7, pp.115418-115427.

[15] Ali Asghari, Mohammad Karim Sohrabi, Farzin Yaghmaee, Task scheduling, resource provisioning, and load balancing on scientific workflows using parallel SARSA reinforcement learning agents and genetic algorithm, The Journal of Supercomputing, (2020), Vol.77, pp.2800-2828, DOI: 10.1007/s11227-020-03364-1

[16] Akm Ashiquzzaman, Tai-Won Um, Jinsul Kim, Energy-Efficient Sensor Calibration Based on Deep Reinforcement Learning, International Journal of Artificial Intelligence and Applications for Smart Devices, (2019), Vol.7, No.1, pp.7-14, DOI: 10.21742/IJAIASD.2019.7.1.02

[17] Ershen Wang, Tao Pang, Yongming Yang, Shujie Zhang, Pingping Qu, GPS Receiver Autonomous Integrity Monitoring Based on Hierarchical Particle Filter, International Journal of Control and Automation, (2016), Vol.9, No.10, pp.303-314, http://dx.doi.org/10.14257/ijca.2016.9.10.28.

[18] Minwoo Ryu, Si-Ho Cha, Context-Awareness based Driving Assistance System for Autonomous Vehicles, International Journal of Control and Automation, (2018), Vol.11, No.1, pp.153-162. 